\title{
HYBRID STEEL ROLL GRADES FOR HOT STRIP MILL LATE FINISHING STANDS*
}

\author{
Mats Söder ${ }^{1}$ \\ Zhi Zhang ${ }^{2}$ \\ Ron Webber ${ }^{3}$
}

\begin{abstract}
The ever increasing demand of high performance rolls in the late finishing stands (F5 - F7) for hot strip mills (HSM) requires better product solutions from roll suppliers. Roll development activities never stop at Union Electric Åkers (UEÅ) in order to meet the requirement. The HYBRID STEEL, a new concept of roll materials especially for the late finishing stands in the HSM, is successfully developed in UEA. This paper reviewed the development of previous ICDP as well as enhanced ICDP (eICDP) roll materials, and, the novel HYBRID STEEL roll grade. Rolls manufactured by this new grade have been used in several European mills. A significant improvement over the enhanced ICDP rolls has been observed.
\end{abstract}

Keywords: Hot Strip Mill; Enhanced ICDP Roll; Hybrid Steel Roll; Late Finishing Work Rolls.

1 Union Electric Åkers, Bruksallen 14, Åkers Styckebruk, Sweden SE-64751, Phone: +46 159321 50. Email: mats.soder@akersrolls.com.

2 Union Electric Åkers, Bruksallen 14, Åkers Styckebruk, Sweden SE-64751, Phone: +46 159321 68. Email: zhi.zhang@akersrolls.com.

3 Union Electric Ákers, 50 Galley Rd, Ancaster, ON, Canada L9G 4T1, Phone: +1-905-512-3525. Email: ron.webber@akersrolls.com. 


\section{INTRODUCTION}

Hot Strip Mill rolling operations have been waiting for improved roll solutions that minimize required roll changes which impact efficiency. Enhanced Indefinite Chill rolls were first introduced back in the mid-1990s. Recent developments have resulted in improved wear characteristics through significant transformation of the iron based graphite and matrix morphology along with adaptation of tool steel based carbides. The application of the enhanced metallurgical properties is characterized, along with the benefits of the extended performance achieved. Hot strip mills are realizing improved operating efficiency during the transition of their entire roll inventories to these break through products.

\section{EVOLUTION OF LATE FINISHING STAND ROLL USE}

The evolution of roll technology has been driven by the consumer. Consumers demand higher quality and cheaper products. This translates into the steel user demanding a better product from the steel Mill (higher strength, thinner, better surface, improved profile and flatness). To achieve this, the Mill Builders have enhanced the Mill design with shifting, bending, more power, CVC, pair cross, just to mention a few of the improvements. This puts great demands on the Mill rolls which have resulted in developments in both the process and product side.

On the process side, static cast rolls were replaced by double pour rolls and then finally, centrifugal cast and CPC methods. Most Hot Strip Mill Finishing Stand rolls are centrifugal cast. The exception being in Japan and various North American Mills, where CPC rolls produced in Japan are used.

There have been few revolutionary changes in roll grades. Prior to the 1970s, ICDP rolls were used in all stands. Adamite gradually replaced ICDP in the early stands. In the early 1970s, after initial setbacks, high chrome iron quickly replaced adamite as they seamlessly provided significant performance improvements. HSS rolls were introduced in the early 1990s. Despite another quantum improvement in performance, their adoption has been slow due to several issues (mainly related to roll cooling).

While these early stand improvements have gone on, little has been done for the delivery stands. The manufacturing of this roll has changed (chill to indefinite chill, static to double pour to centrifugal pour) but the grade remained the same for over 60 years. In the early 1990s, British Rollmakers made an improvement by adding powder containing carbides to the liquid melt. In 1997, Åkers patented a technique to do this without adding powder as the powder tended to segregate due to density differences.

The new generation of enhanced carbide ICDP rolls resulted in a performance improvement of up to $25 \%$, well short of the 2 to 4 times improvement with HSS rolls over high chrome iron rolls. Historically it had been common to have an early stand roll change half way through the campaign as the ICDP rolls outlasted the adamite / high chrome iron rolls. Currently, HSS rolls can be used for 2 to 7 campaigns. Since, with few exceptions, rolls are changed in parallel, it takes as long to change one stand as it does to change all 7 . For all the other benefits of HSS rolls (lower roll cost, better surface, better profile and flatness, better gauge control), their longer campaigns do not result in increased Mill capacity. Thus, there is a need for latter stand rolls with significantly better performance in order to achieve productivity improvements by better matching the campaign length of HSS (or high chrome iron).

\section{DEVELOPMENT OF HYBRID STEEL ROLLS}

The milestones for delivery stand roll grade at Union Electric Åkers chronologically are the ICDP, Enhanced ICDP and the newest HYBRID STEEL. Their performance is ever increasing, too. The IC rolls in the early stage represent the old design of Ni-hard material and focus has been on the creating of a mass of cementite over the matrix to generate higher hardness and better wear resistance compared with white iron. Later on, the MC type of carbide was first introduced into Enhanced ICDP rolls to improve the performance. 
Presently, the most recent HYBRID STEEL roll material development aims at introducing even more $\mathrm{MC}$ type of carbide and other types to achieve a better balance of the amount of cementite, MC type carbides and graphite which will lead to a homogeneous distribution of all phases. By this concept, a large amount of elements that promote the formation of MC type of carbide are used. Figure 1 shows the microstructural comparison between the ENHANCED ICDP and the HYBRID STEEL material.

The percentage or area fraction of cementite in the original ENHANCED ICDP grade is around $35-40 \%$. It is seen by the image that the cementite still predominate in the newest HYBRID STEEL grade, however, its total amount is about only $10 \%$. A higher amount MC carbide forming elements will postpone the formation of cementite. Well distributed $\mathrm{MC}$ carbides isolate the later formed cementite. The MC carbide content is similar to that found in normal HSS rolls.

In the early stage of development of this new grade, the toughness was low. It was found that the hardness was too high, even after several heat treatments. Numerous calculations and laboratory trials indicate good correlation with carbide, graphite and matrix balance and a dependable prediction model has been developed. The uniquely designed composition allows a different sequence of solidification which prioritized the graphite formation. Figure 2 shows a Scheil model calculation comparison between the ENHANCED ICDP and HYBRID STEEL grades.

\section{Carbides in HYBRID STEEL (x100) Carbides in eICDP (x100)}
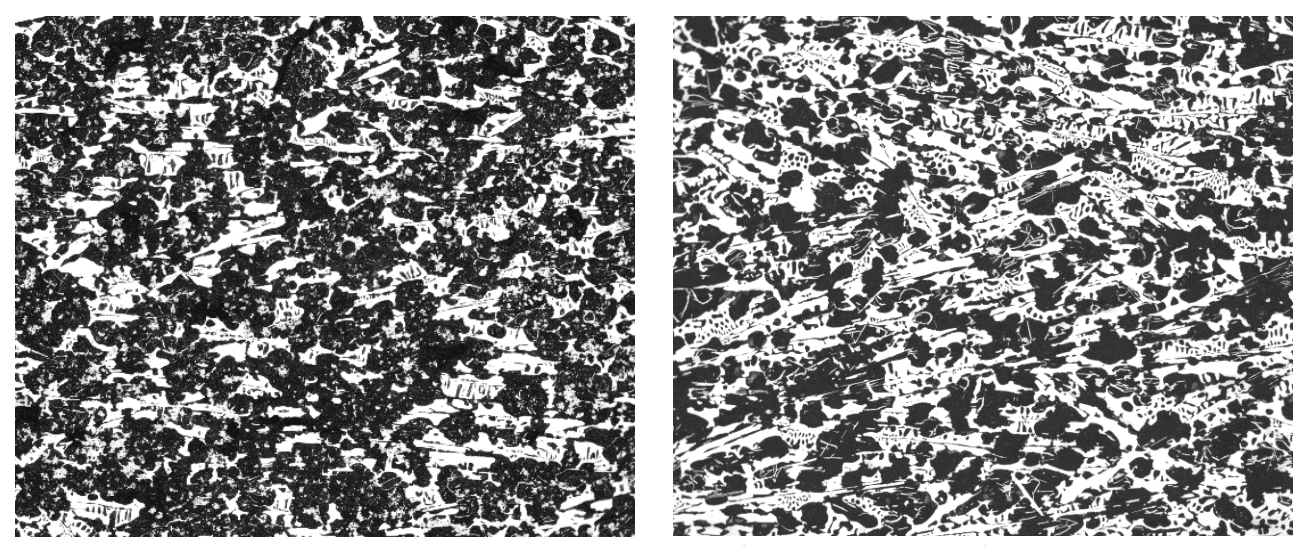

Fig. 1 Microstructure difference between HYBRID STEEL and eICDP rolls (x100)
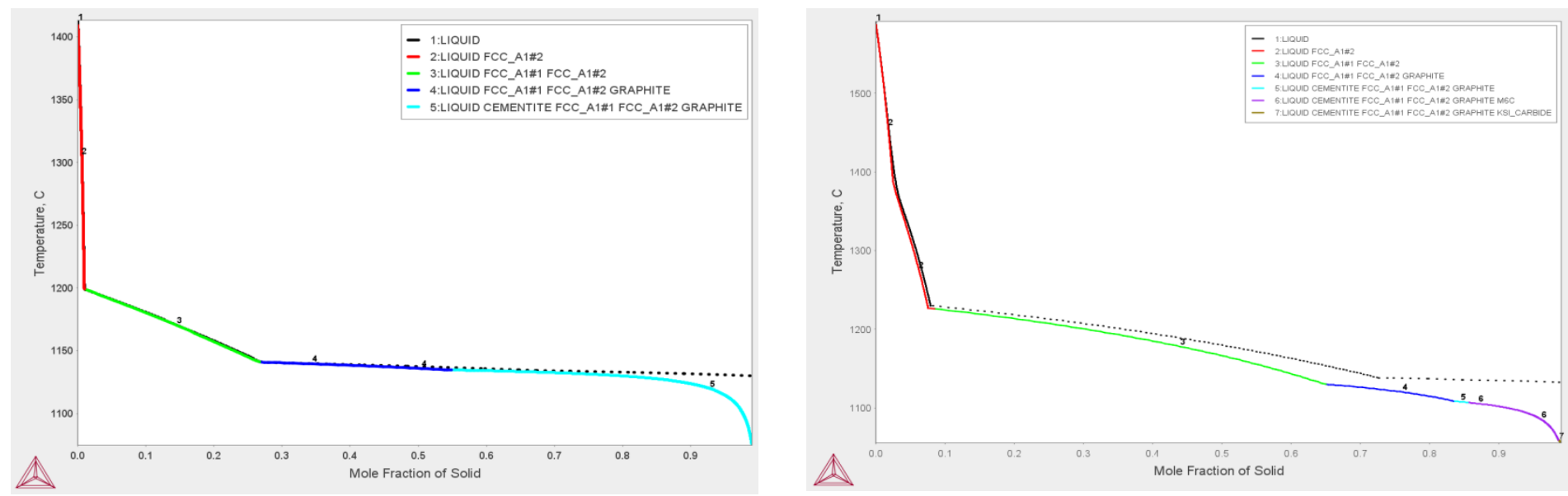

Fig. 2 Scheil prediction of phases during solidification (left: Enhanced ICDP; right: HYBRID STEEL) 
Oxidation during hot rolling has an important effect on roll performance and product surface quality, especially for the HSS grades used in the early finishing stands in the mill. Since this new grade is highly alloyed and it is similar to a standard HSS composition, its oxidation behavior was of concern. Thus, an oxidation simulation has been carried out to investigate the cumulative effects of water vapor and air on the formation of oxide scales at high temperatures.

Figure 3 shows the oxidation behavior of these two alloys. Experiments were performed at $900^{\circ} \mathrm{C}$ under an atmosphere of mixture of air and water vapor, which was introduced at a controlled rate of $0,2 \mathrm{l} / \mathrm{min}$. The size of samples is standard $(20.5 \times 8.5 \times 6.5 \mathrm{~mm})$. In this case, the sample surface will be oxidized and the entire sample weight gradually increases. The gain of weight is therefore recorded continuously. Each sample was tested for 60 minutes.

The HSS material is easily oxidized in the very beginning stages $(<200 \mathrm{~s})$. This may be due to the higher percentage of iron based matrix compared with the IC rolls. However, the high $\mathrm{Cr}$ content in the HSS grade does not prevent the oxidation process as compared to an IC grade in this simulation. HYBRID STEEL oxidizes slowest among the three materials. This is due to a much higher amount of alloying elements, such as Mo, W, V and Cr dissolved in the matrix, which promote the formation of a tight and protective oxide layer. This layer is formed in the initial periods and then retards the oxidation process. This phenomenon is explained in Figure 4.

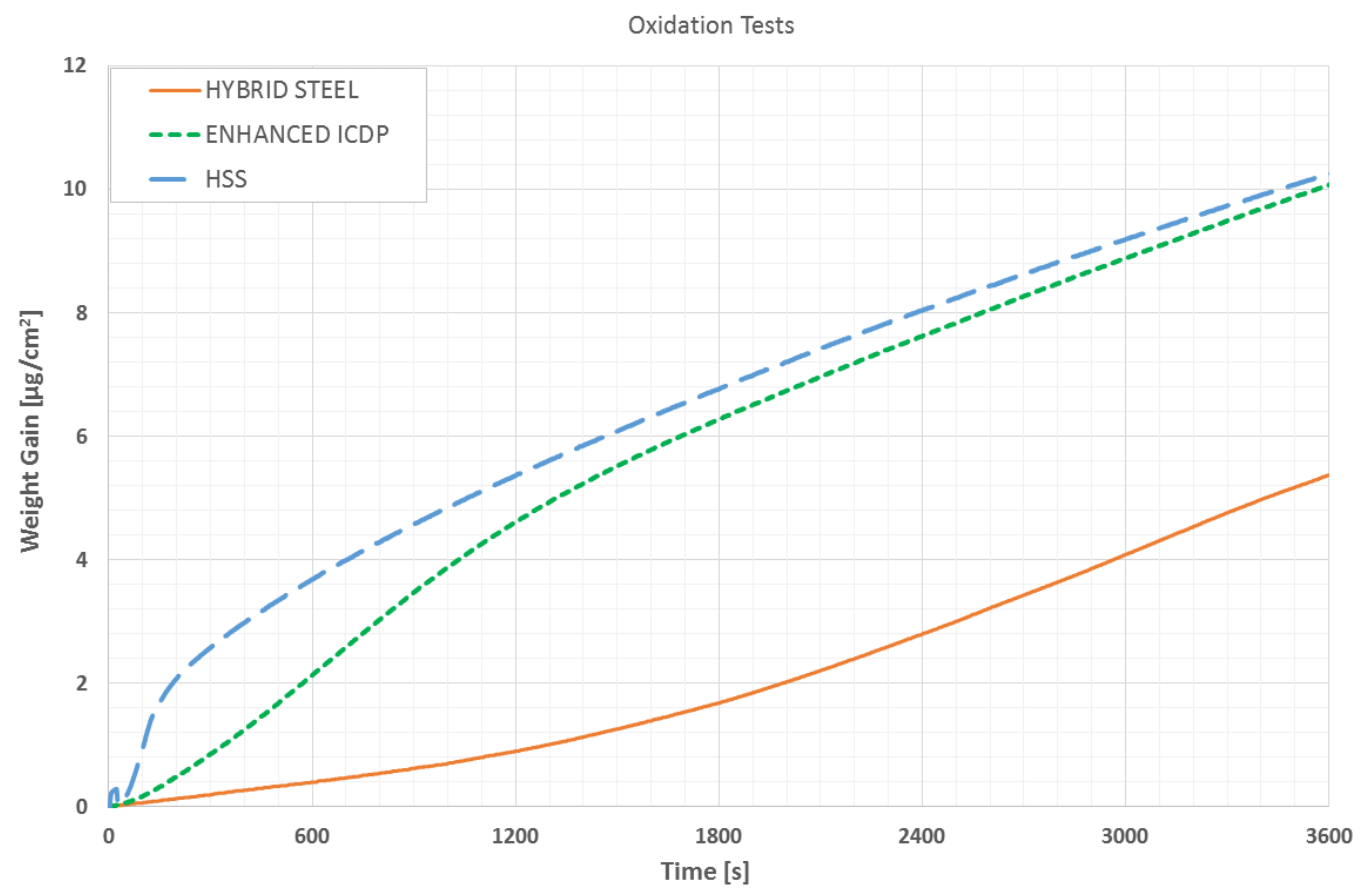

Fig. 3 Oxidation layer of HYBRID STEEL 


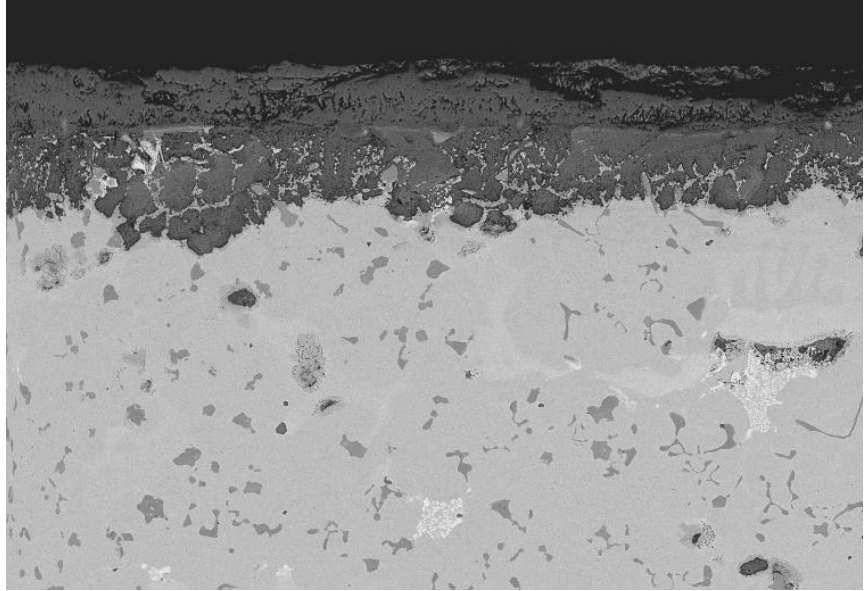

$x 50$

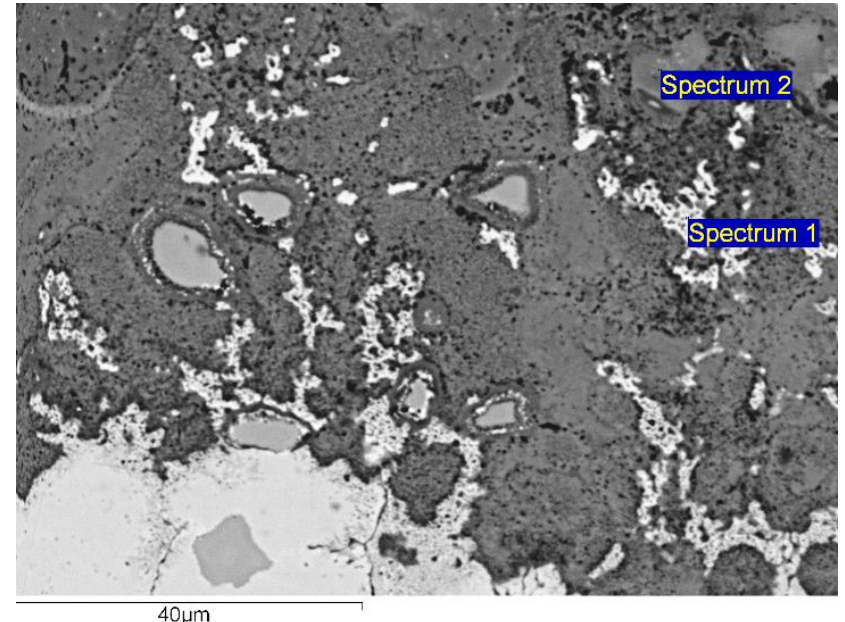

$\mathrm{x} 1000$

Fig. 4 The development of the oxide scale; The oxidation penetrate through the grains and the last oxidized regions are at the grain boundaries. Spectrum-1 in the right image shows that these regions are with high amount of $\mathrm{Si}$ and $\mathrm{Ni}$, which reduce the oxidation process.

Retained austenite can be problematic for the materials applied to rolls. An alpha value measured by using a ferritiscope at Union Electric Åkers is a fast method to predict the approximate level. In order to quantify a true amount of retained austenite in the matrix however, the Bruker D8 X-ray diffractometer is employed by using $\mathrm{Cu}$-ka radiation. Figures 5 and 6 depict the diffraction patterns and the details of the FCC [311] peak.

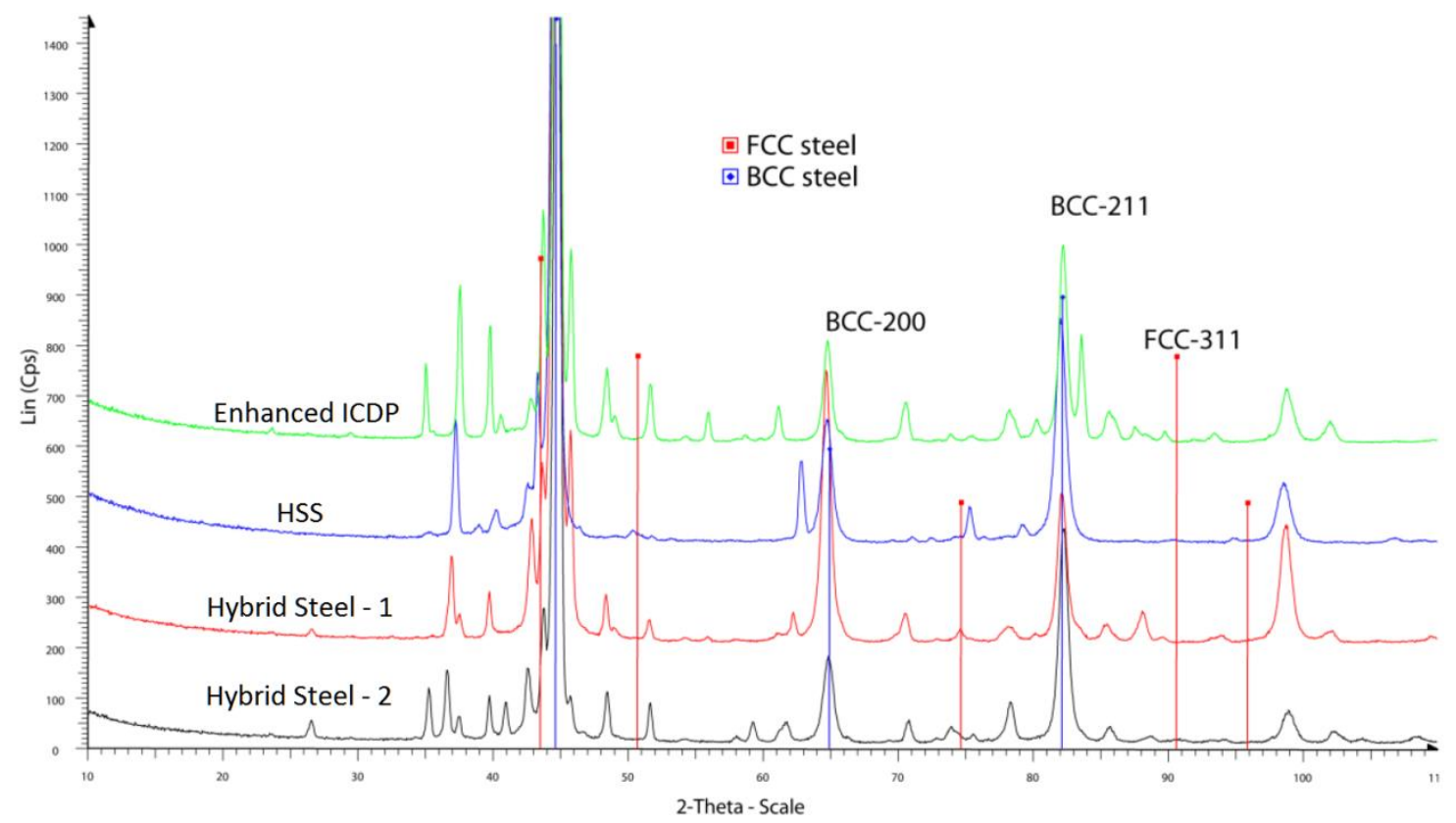

Fig. 5 Diffraction patterns of the investigated samples. The peaks used for quantification of retained austenite volume are indicated (BCC-200, BCC-211 and FCC-311). As seen in the diffractograms multiple peaks were found in addition to the BCC and FCC phases. These are probably originating from carbides and the volume of these were not taken account in the quantification. 


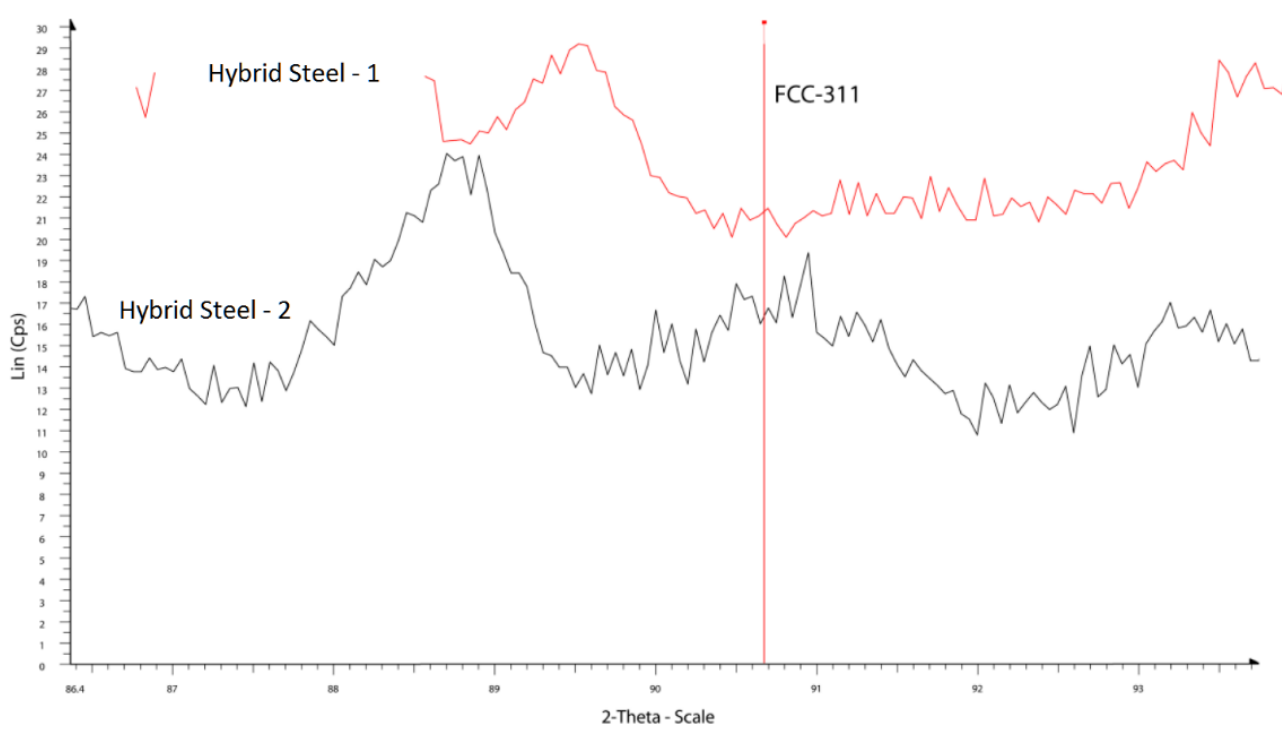

Fig. 6 Detail at the position for the FCC peak 311. Intensity was found for HYBRID STEEL-1 whereas no intensities were found for samples HYBRID STEEL-2. For the quantification of the retained austenite, volume integrated intensities were used.

The amount of retained austenite was calculated by using the intensity of the FCC peak [311] compared to the total (FCC + BCC) with an intensity factor that takes each peak into account. No volume of carbides or other precipitates were taken account. Results shows that the total amount of retained austenite is less than $1 \%$ which means the new type of HYBRID STEEL roll is very safe to use.

The residual stress in the new roll grade was examined to ensure that it is a low level. A destructive measurement was done in the middle of the roll barrel. In this method, a specially configured strain gage rosette is attached to the surface of the roll barrel; and a small, shallow hole with a diameter of less than $2 \mathrm{~mm}$ and a depth of $1.3 \mathrm{~mm}$ is introduced into the structure, through the center of the gage, with a precision drilling apparatus. Strains in the immediate vicinity of the hole are measured, and the relaxed residual stresses are computed from these measurements. The strain in three gages can be measured directly from the equipment. Then, the maximum and minimum stress can be calculated by using the following equations:

$$
\sigma_{\max / \min }=\frac{\varepsilon_{1}+\varepsilon_{3}}{4 A} \pm \frac{\sqrt{\left(\varepsilon_{3}-\varepsilon_{1}\right)^{2}+\left(\varepsilon_{3}+\varepsilon_{1}-2 \varepsilon_{2}\right)^{2}}}{4 \mathrm{~B}}
$$

Where the coefficient $A$ and $B$ can be determined by using:

$A=-\frac{1+v}{2 E}\left(\frac{1}{r^{2}}\right), \mathrm{B}=-\frac{1+v}{2 \mathrm{E}}\left[\left(\frac{4}{1+v}\right) \frac{1}{\mathrm{r}^{2}}-\frac{3}{\mathrm{r}^{4}}\right], r=\frac{D_{0}}{D}$

Do $=$ diameter of drilled hole

$\mathrm{D}=$ gage circle diameter

Figure 7 shows the stress at each drilling of $0.13 \mathrm{~mm}$ and it is shown that the residual stress trends to stabilize under $1 \mathrm{~mm}$ depth. The maximum stress is below $200 \mathrm{MPa}$, which is in the normal range for ICDP rolls. 


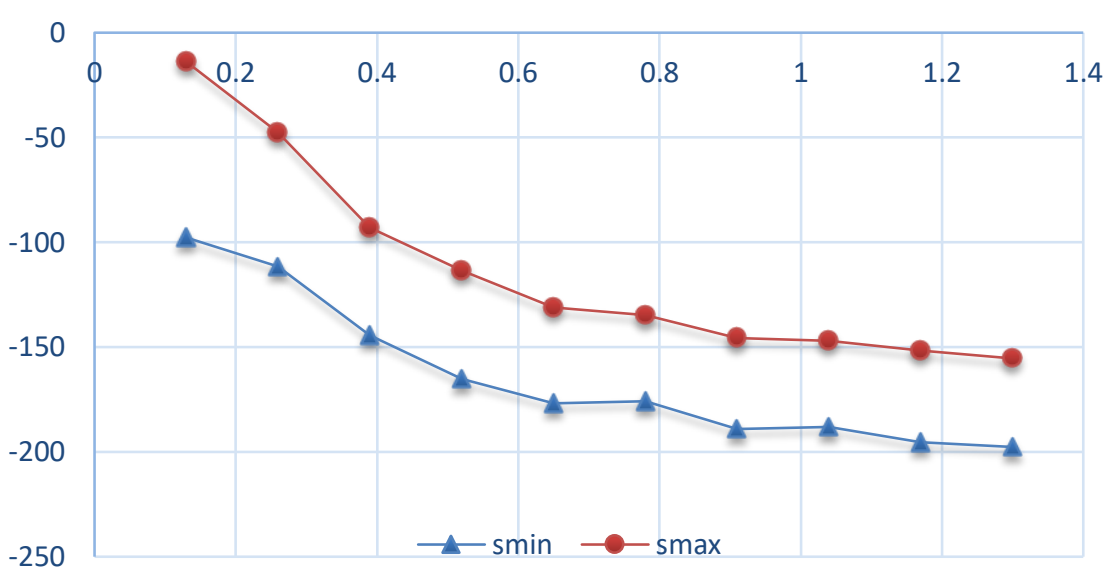

Fig. 7 Residual stress measurement on the middle barrel of a HYBRID STEEL roll (MPa) to a depth of $1.3 \mathrm{~mm}$. Max stress is less than $200 \mathrm{MPa}$

Recently, many mills are equipped with eddy current equipment to monitor the roll surface after roll grinding. The results of eddy current scanning may vary between different roll grades. Figure 8 shows the eddy current results of a HYBRID STEEL roll, which is measured by using LISMAR (2015 model) The same threshold is used as for normal ICDP and elCDP rolls $(0.2)$.
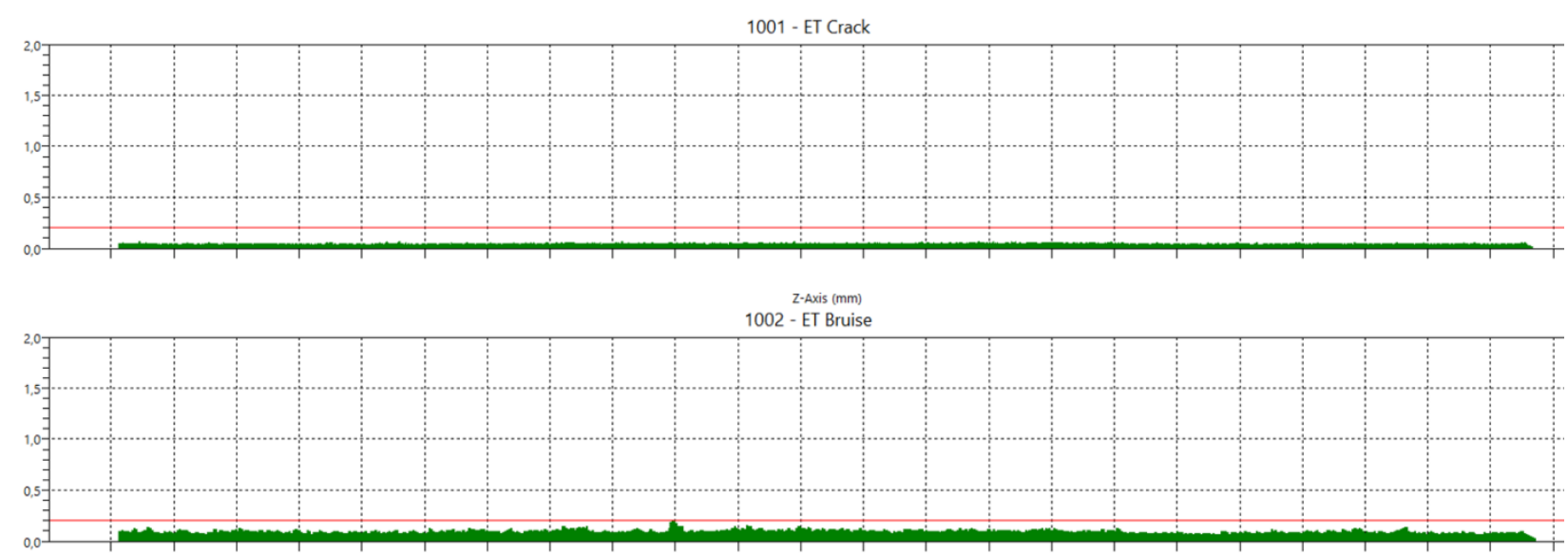

z-Axis (mm)

Fig. 8 Eddy Current testing of crack and bruise at the surface of a HYBRID STEEL roll

\section{INDUSTRIAL RESULTS}

Trials have been conducted at a number of mills. A significant improvement in performance has been attained in all trials. Results from two mills are shown below: 


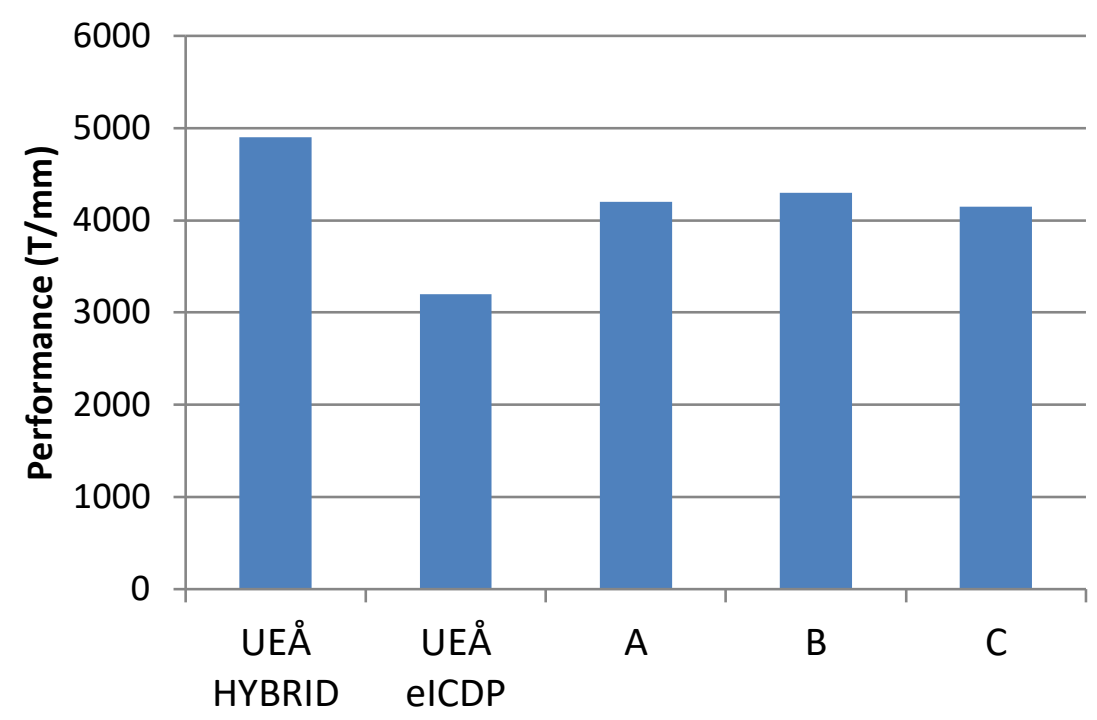

Fig. 9 UEÅ HYBRID STEEL Roll Performance versus Competitor's similar roll types and the regular UEÅ eICDP

Figure 9 shows the results from a conventional HSM and includes mill incidents. The performance of the UEA HYBRID STEEL roll is just over $50 \%$ better than that of the UE elCDP. The HYBRID roll was used in the last 3 finishing mill stands.

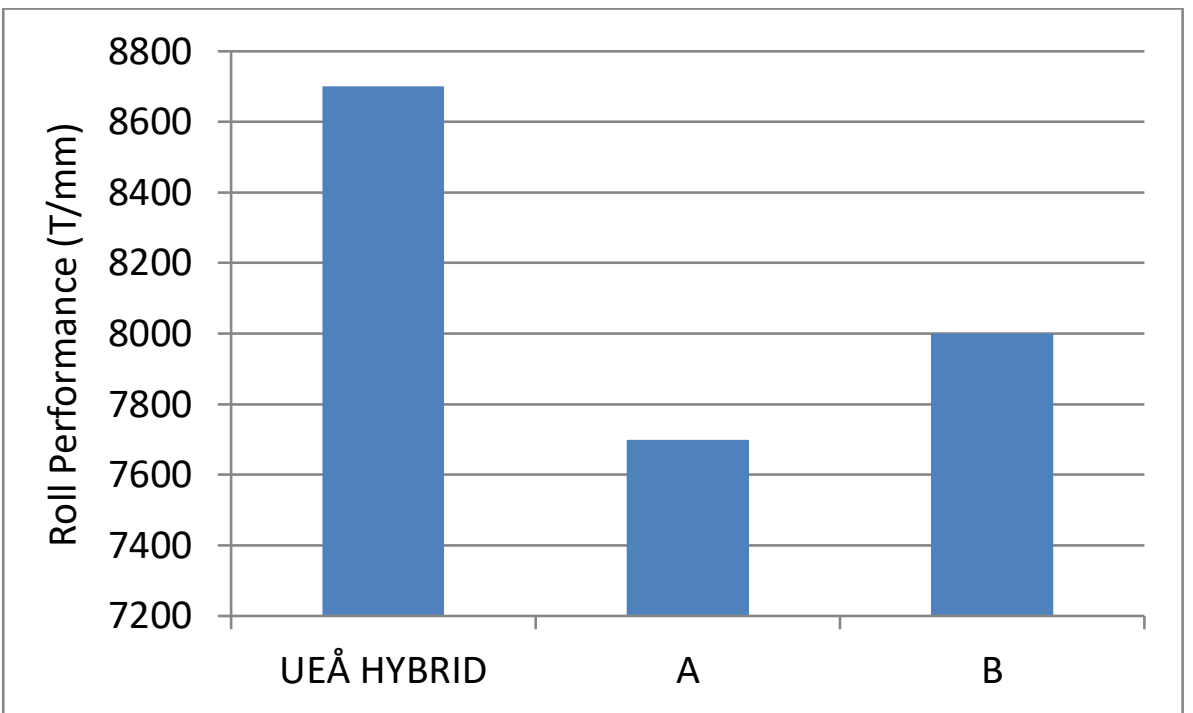

Fig. 10 HYBRID STEEL performance versus competitor's performance from the same type of roll.

Data from another conventional Hot Strip Mill is shown in Figure 10. In this case, the performance data for eICDP rolls was unavailable.

In all cases, the HYBRID STEEL roll outperforms eICDP by at least $25 \%$ and up to $50 \%$. It also performs better than, or as well as similar type of rolls from competitors. Some mills are now using Hybrid rolls for double campaigns.

It is seen from Fig. 11 that rolls present a very good and uniform surface aspect, smooth and clear grey in a condition of after rolling. 

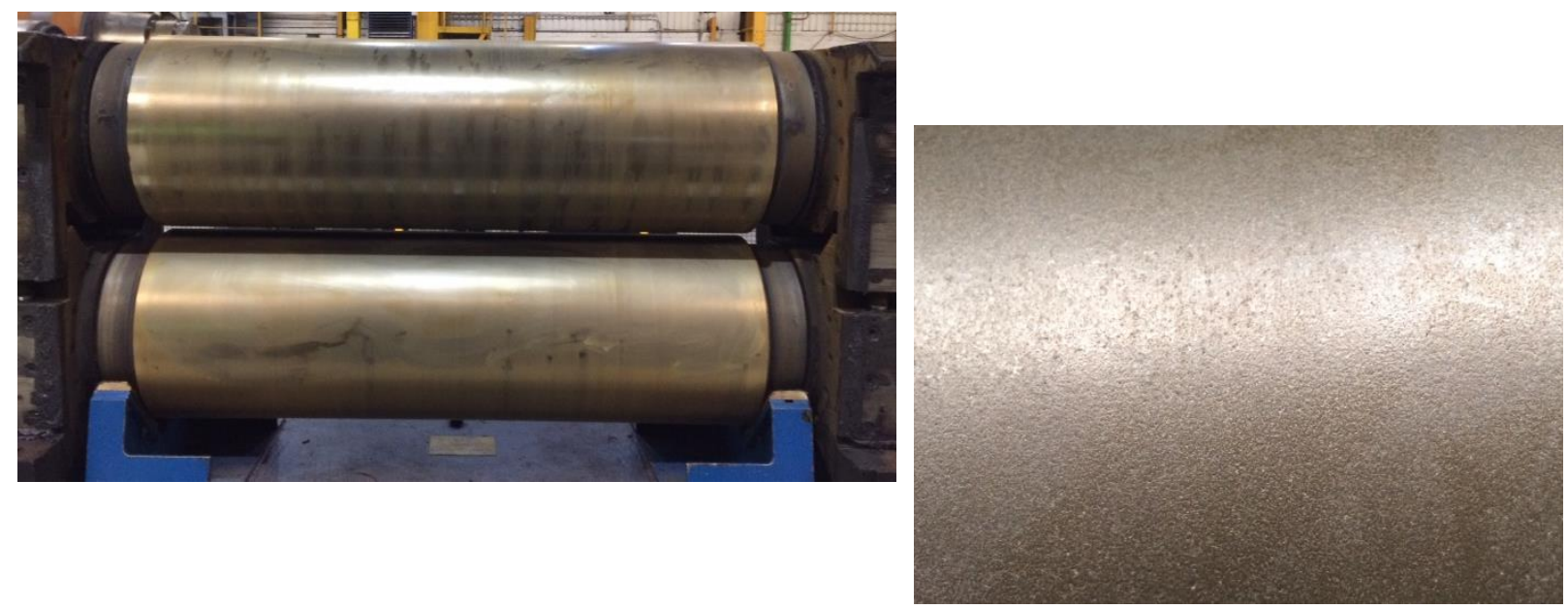

Fig. 11 Roll surface conditions after rolling

The HYBRID STEEL roll maintains the advantages that have resulted in the ICDP roll being the preferred latter stand roll for over 70 years: resistance to crack propagation and resistance to strip welding.

\section{CONCLUSIONS}

The Union Electric Åkers HYBRID STEEL roll is a new generation of roll that provides a significant improvement in performance. This enables the HSM to lower roll costs while also improving the productivity of the mill by extending rolling campaigns. By adapting high speed tool steel based carbides to an iron based graphite morphology, the campaign lengths of entry (HSS) and delivery stands can be closer matched. 